\section{Neuer Monolith in der Inhalationstherapie}

D ie Evolution geht weiter - so kommentierte Dr. Wolfgang Fleischer, Bereichsleiter Forschung und Entwicklung bei Mundipharma, die Entwicklung im Bereich der Pulverinhalationssysteme: Waren sie bei ihrer Einführung Mitte der 70er-Jahre eine zwar umweltfreundliche, aber nur für einen sehr begrenzten Patientenkreis geeignete Therapieoption, schicken sie sich nun an, eine vollwertige Alternative, wenn nicht gar ein Ersatz für die FCKW-getriebenen Dosieraerosole zu werden.

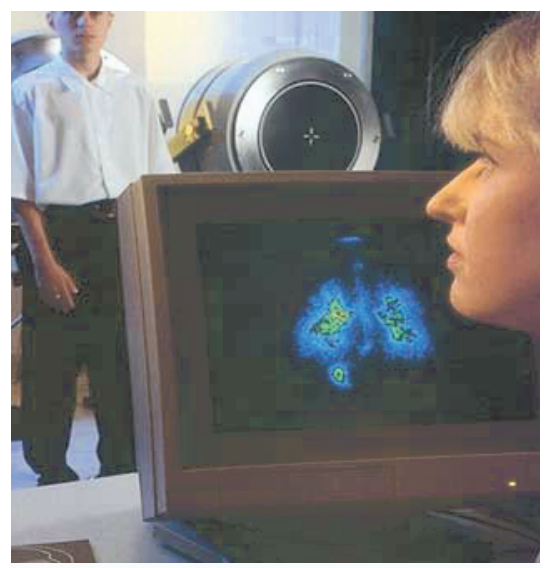

Auch ein geringer inspiratorischer Fluss führt zu einer ausreichenden bronchialen Deposition - Szintigrafie nach Inhalation mit dem MAGhaler ${ }^{\circledR}$

Großes Manko der Pulverinhalatoren sind die physikalischen Eigenschaften des Pulvers selbst, das feuchtigkeitsempfindlich ist und zur Agglomeration neigt. Außerdem ist zur Mobilisation und Aspiration des Wirkstoffs ein gewisser Mindestatemfluss notwendig, was z. B. die Verwendbarkeit bei Kindern einschränkt. Beide Nachteile vermeidet das neuartige Inhalationssystem MAGhaler ${ }^{\circledR}$ weitgehend: Das Arzneimittel liegt nicht mehr in loser mikronisierter Form vor, sondern ist zu einem Monolithen verpresst. Erst unmittelbar beim Inhalationsvorgang schabt eine mechanisch angetriebene Keramikfräse die benötigte Dosis ab. So lassen sich ohne die Gefahr einer Agglomeration mit immer gleicher Präzision 200 bis 300 Einzeldosen erzeugen.
Die frisch abgeriebenen Partikel sind zudem bereits mobilisiert, auch ein geringer inspiratorischer Fluss führt also zu einer ausreichenden bronchialen Deposition. In der Tat zeigten szintigrafische Untersuchungen, dass mit einem Atemzugsvolumen von $30 \mathrm{l} / \mathrm{min}$ eine ähnlich gute pulmonale Wirkstoffverteilung erzielt wird wie mit $60 \mathrm{l} / \mathrm{min}$.

Dr. Josef Lecheler vom CJD Asthmazentrum Berchtesgaden berichtete über erste praktische Erfahrungen mit dem neuen Inhalator bei Kindern. Im direkten Vergleich mit einem Dosieraerosol zeigte sich, dass beide Systeme in etwa gleich wirksam und auch gleich verträglich sind. Die Akzeptanz des MAGhalers ${ }^{\circledR}$ sei bei den beteiligten Kindern sogar größer gewesen, so Lechler: „Das ist insofern besonders bemerkenswert, als Kinder in früheren Studien immer das Dosieraerosol bevorzugten." Man darf also auf weitere Studienergebnisse gespannt sein.

Der MAGhaler ${ }^{\circledR}$ ist seit August mit dem Wirkstoff Budesonid als Respi$\operatorname{cort}^{\circledR}{ }^{\circledR A G h a l e r}{ }^{\circledR}$ in Deutschland erhältlich.

Symposium „MAGhaler ${ }^{\circledR}$ - das andere Pulverinhalationssystem" im Rahmen des 42. Kongresses der Deutschen Gesellschaft für Pneumologie, Jena 2001. Veranstalter: Mundipharma

\title{
Immuntherapie verbessert Lebensqualität
}

n der Beurteilung des Therapieerfolges kommt der Lebensqualität heute ein hoher Stellenwert zu. Neben der Verbesserung von klinischen Symptomen und Laborparametern liefern vor allem die Daten zur Lebensqualität einen wichtigen Hinweis über die Effektivität einer Behandlungsmethode. Die verbesserte Lebensqualität ist darüber hinaus entscheidend für die Compliance der Patienten.

In einer neuen Studie mit 1.257 Atemwegsallergikern wurden Daten zur Lebensqualität vor und nach einer einjährigen spezifischen Immuntherapie (SIT) mit molekular standardisierten Gräser- und Roggenpollenallergenen erhoben. Eingesetzt wurde dabei u. a. der krankheitsspezifische Rhinitis-Lebensqualitätsfragebogen nach Juniper. $\mathrm{Zu}-$ gleich wurde die Compliance unter der SIT anhand eines Therapieprotokolls beurteilt.

Wie der wissenschaftliche Leiter der Studie, Prof. Karl Christian Bergmann, Bad Lippspringe, ausführte, ergaben sich sowohl bei der Lebensqualität wie auch der Compliance positive Resultate. So verbesserte sich der mittlere Score des krankheitsspezifischen Lebensqualitätsfragebogens signifikant von 2,88 vor der SIT auf 1,67 nach dem ersten Behandlungsjahr. Die deutlichs- ten Verbesserungen ergaben sich für die Nasen- und Augensymptome sowie für den Schlaf und die Arbeitsleistung. Auch durch die Krankheit beeinträchtigten Aktivitäten wurde nach der einjährigen Immuntherapie wieder in verstärktem Umfang nachgegangen.

Besonders erfreulich, so Bergmann, waren auch die Ergebnisse zur Compliance: Mehr als 93\% der Patienten setzten die Immuntherapie nach dem ersten Behandlungszyklus fort, lediglich bei $1,2 \%$ gab der Arzt ausdrücklich "mangelnde Compliance" als Abbruchgrund an. Diese Rate liegt deutlich niedriger als die in der Literatur berichteten Raten unter einer symptomatischen Therapie.

Die in dieser Studie gefundenen Ergebnisse decken sich weitgehend mit denen aus US-amerikanischen Studien. Und dort ergab sich noch ein weiterer interessanter Befund: Wurde die SIT durchgängig in einer allergologischen Facharztpraxis durchgeführt, war die Compliance wesentlich höher als bei einer Weiterbehandlung durch nicht spezialisierte Ärzte.

Satellitensymposium anlässlich des 20. Kongresses der European Academy of Allergology and Clinical Immunology, Berlin 2001. Veranstalter: ALK Abelló 